\title{
Construção de um colorímetro de baixo custo para uso em produtos agrícolas
}

\author{
Jedman Dantas Mota', Alexandre José de Melo Queiroz' \\ Rossana Maria Feitosa de Figueirêdo', Karla dos Santos Melo de Sousa*2 \\ 'Universidade Federal de Campina Grande, Campina Grande, PB, Brasil \\ 2Universidade Federal do Vale do São Francisco, Petrolina, PB, Brasil \\ *Autor correspondente; e-mail: karla.smsousa@univasf.edu.br
}

\section{Resumo}

Com este trabalho objetivou-se construir um colorímetro de baixo custo, cujo princípio de funcionamento consiste em se determinar a refletância das amostras em relação a um padrão de branco e calcular as coordenadas do sistema de cor L*a*b* com uso das equações propostas pela CIE (Comission Internationale d'Eclairage). O aparelho foi construído em bloco compensado de madeira e tem, como fonte de iluminação, 21 LED's divididos em 7 grupos conforme a cor. 0 sensor tem resposta aproximada à do olho humano sendo ideal para aplicações em fotometria. O desempenho do instrumento foi comparado com um modelo comercial, do qual se obteve uma relação linear entre as coordenadas cromáticas dos dois equipamentos, com a calibração realizada com 60 amostras de pó colorido.

Palavras-chave: análise instrumental, cor, reflectância

\section{Colorimeter construction of a low cost for use in agricultural products}

\begin{abstract}
This work aimed to build a low cost colorimeter was constructed, whose operation principle consists of determining a sample reflectance respecting a White Standard and compute the coordinates of $L^{*} a^{*} b^{*}$ color space using the CIE (Comission Internationale d'Eclairage - International Illumination Commission) equations. The equipment was constructed with wood pressed, and operates with 21 LED's divided into 7 groups according to their color. The sensor has a similar response to the human eye is ideal for applications in photometry. The performance of the instrument was compared to a commercial model CR-10, in which a linear relationship was obtained between the chromaticity coordinates of the two units, with the calibration performed with 60 samples of colored powder.
\end{abstract}

Keywords: Instrumental analysis, Color, Reflectance 


\section{Introdução}

A aceitação ou rejeição de alimentos pelo consumidor depende de vários parâmetros, dos quais destaca-se a cor (Miguel et al., 2009); na indústria não é diferente, porque o produto agrícola deve sair do campo no momento certo para que possua e mantenha os atributos de qualidade ideais para o processamento. Quando o objetivo é a exportação, o produto agrícola deve ser colhido em um momento preciso, conforme a distância e o tempo entre a colheita e o consumo, de forma a completar sua maturação no período de transporte e chegar ao destino detendo as qualidades exigidas pelo comprador.

A classificação de cor pode ser feita por vários métodos; no caso de alimentos, o mais utilizado é o subjetivo, em que o consumidor se baseia na sua própria interpretação. Outro método bastante empregado é o comparativo, baseado no qual uma cor é comparada, sob condições controladas, com um padrão. Podemos ainda citar o método direto, bem menos empregado, em que a cor é classificada com base nos fenômenos físicos que ocorrem na interação energia-matéria; esta classificação, por sua vez, se dá com uso de equipamentos específicos.

Existem vários sistemas de medição de cor: o sistema RGB; Sistema de Munsell; sistema CIE (1931) X, Y e Z; Sistema L*, a*, b* (CIE, 1976 ou CIELAB) e Sistema $L, a, b$ de Hunter (Faraoni et al., 2008).

O espaço de CIELab foi até agora o mais largamente e usado para apresentar cores, pois possui espaço dimensional. A escala neutra fica situada no centro, sendo que o espaço L (luminosidade) varia entre zero (preto) e 100 (branco), os valores de a e b (coordenadas de cromaticidade) variam de -a (verde) até +a (vermelho), e -b (azul) até +b (amarelo) (Luo, 2006). Estes parâmetros são determinados através de colorímetro, este utiliza a técnica de avaliação das cores refletidas pelos objetos e através de uma célula fotossensível e um medidor indicam os parâmetros em uma escala numérica (Leão et al., 2006)

O uso corriqueiro de colorímetros comerciais em unidades produtivas agrícolas tem como impedimento o custo relativamente elevado de tais equipamentos para produtores, agroindústrias e laboratórios de pequeno porte. A disponibilidade de colorímetros de baixo custo possibilitaria a utilização da análise instrumental de cor como meio de avaliação rotineira de diversas matérias-primas sob condições variadas, como degradações causadas pelo efeito do tempo de armazenamento, alterações provocadas por aquecimentos, luminosidade, efeitos do frio, além de determinação instrumental de estádio de maturação em frutas.

Este trabalho foi realizado com o objetivo de se construir um colorímetro de baixo custo e comparar os resultados obtidos com um colorímetro comercial.

\section{Material e Métodos}

A etapa experimental foi desenvolvida no Laboratório de Armazenamento e Processamento de Produtos Agrícolas da Unidade Acadêmica de Engenharia Agrícola da Universidade Federal de Campina Grande, em Campina Grande, Paraíba e na Unidade de Pesquisa e Demonstração em Alimentos do Centro de Formação de Tecnólogos da Universidade Federal da Paraíba, em Bananeiras, Paraíba.

Construiu-se o protótipo de colorímetro utilizando-se 21 diodos emissores de luz (LED) com sete características espectrais diferentes (três diodos para cada cor) e um fotodiodo cuja resposta espectral está situada em todo o espectro visível. O protótipo foi usado na determinação das cores de 60 amostras coloridas artificialmente. Leituras de cor das mesmas amostras foram realizadas com um colorímetro comercial, da marca Minolta, modelo CR-10 e compararam-se os resultados obtidos com ambos os instrumentos.

\section{Construção do colorímetro}

O colorímetro foi construído em bloco compensado de madeira de $15 \mathrm{~mm}$ de espessura,

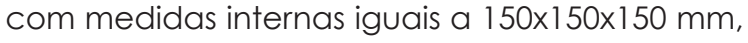
com pintura interna e externa de tinta preta fosca, dotado de orifício no centro da parte superior para passagem da guia metálica e porta lateral de acesso. A guia metálica se 
compunha de um tubo de alumínio de $10 \mathrm{~mm}$ de diâmetro interno com escala graduada. $\bigcirc$ ponto de referência da escala era composto de placa metálica com furo central e foi preso

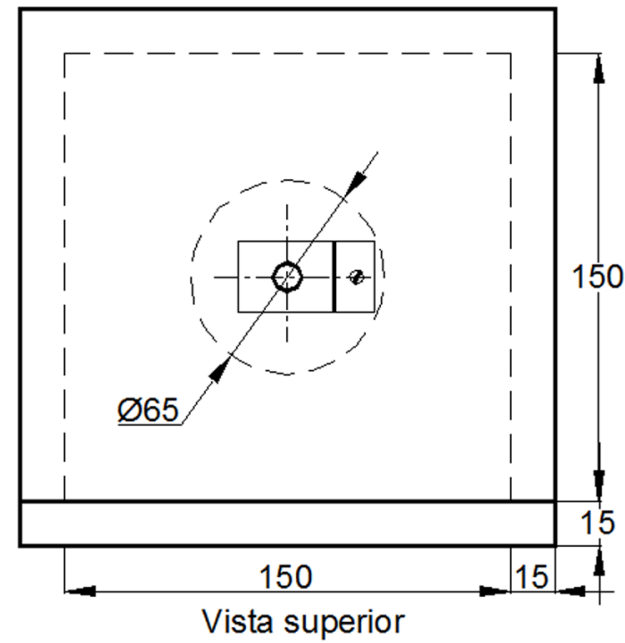

Figura 1. Esquema do protótipo de colorímetro.

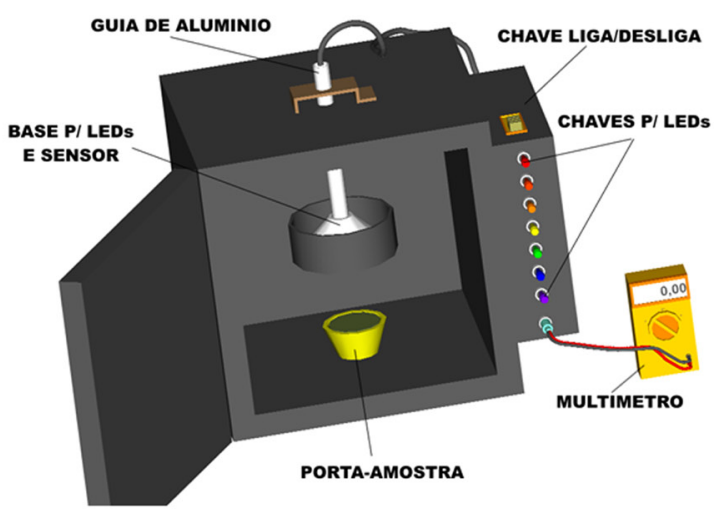

Figura 2. Esquema visual do protótipo de colorímetro.

fontes de radiação controladas por chaves e fotossensor com condicionador de sinal. O princípio básico de funcionamento consiste em medir a variação da fotocorrente provocada pela variação da intensidade luminosa sobre o sensor. A fonte de radiação é formada de 21 LED's de alto brilho de $5 \mathrm{~mm}$ de diâmetro, divididos em 7 grupos, conforme a cor. Tem-se na Tabela 1, a referência e o comprimento de onda dominante de cada LED.

Dispuseram-se os LED's de cada cor específica circularmente, em torno da guia metálica em um suporte com forma de cone, dotado, na base, de lâmina transparente, na parte superior da caixa. Pode-se observar, nas Figuras 1 e 2, os detalhes da construção do protótipo.

O circuito eletrônico é formado de

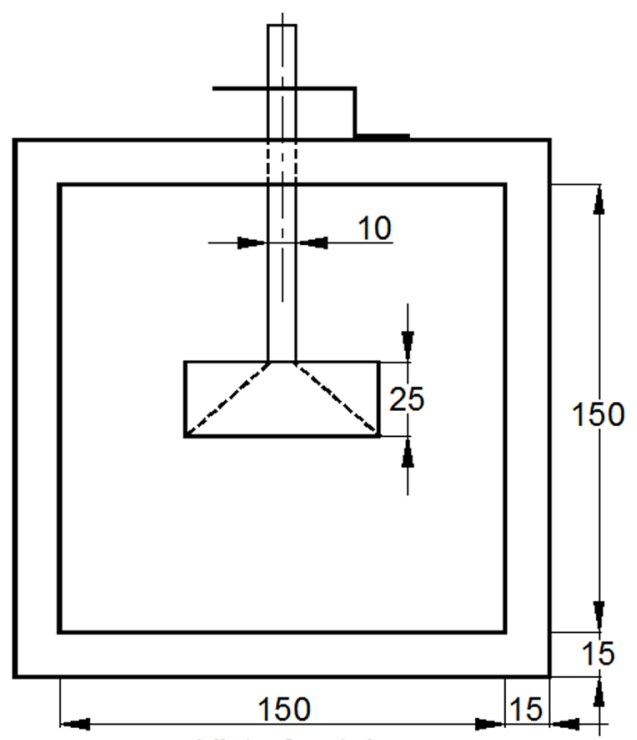

Vista frontal

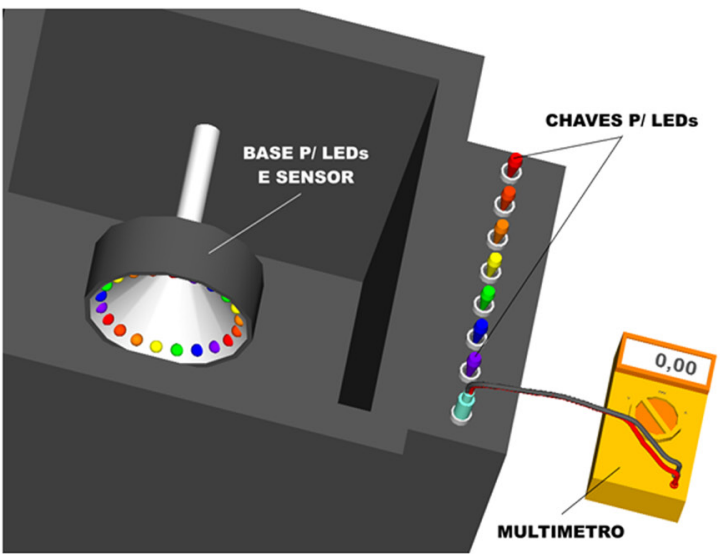

na qual foi colocado um filtro dispersor da marca Cinegel® número 3027, fabricado pela Rosco $^{\circledR}$, para que a iluminação sobre a amostra fosse completamente difusa, ou seja, livre de incidência direta. O diagrama de blocos do colorímetro e a disposição dos LED's podem ser vistos na Figura 3.

O ângulo entre LED's de mesma cor foi de $120^{\circ}$ e estes foram conectados a uma mesma chave, procedimento adotado para que a amostra fosse iluminada uniformemente; desta forma, o equipamento foi dotado de 7 chaves que podem ser acionadas independentemente aumentando, assim, sua versatilidade. O 
fotossensor foi inserido na parte interna da guia metálica a uma distância fixa por onde também se passaram os fios para conexão dos LED's. Utilizou-se o OSD15-E como fotossensor. Este dispositivo apresenta resposta espectral aproximada ao olho humano, pois é dotado de filtro de correção de cor; possui uma área ativa de $15 \mathrm{~mm}^{2}$ e responsividade típica de $15 \mathrm{nA} / \mathrm{lux}$ com tempo de resposta típico de 12 ns.

Tabela 1. LED's usados no colorímetro.

\begin{tabular}{ccr}
\hline LED & Comprimento de onda dominante $\mathbf{( n m )}$ & Referência comercial \\
\hline Violeta & $405 \pm N C$ & OPL155UV0C-20 \\
Azul & $470 \pm 30$ & OPL155SPB16C-30 \\
Verde & $525 \pm 35$ & OPL155PG0C-25 \\
Amarelo & $589 \pm 15$ & OPL155UYOC-25 \\
Laranja & $605 \pm N C$ & OPL155SO2C-15 \\
Âmbar & $615 \pm 17$ & OPL155UA15C-15 \\
Vermelho & $624 \pm 20$ & OPL155SUR15C-30 \\
\hline NC - Não consta no manual do fabricante
\end{tabular}

CONDICIONADOR DE SINAL

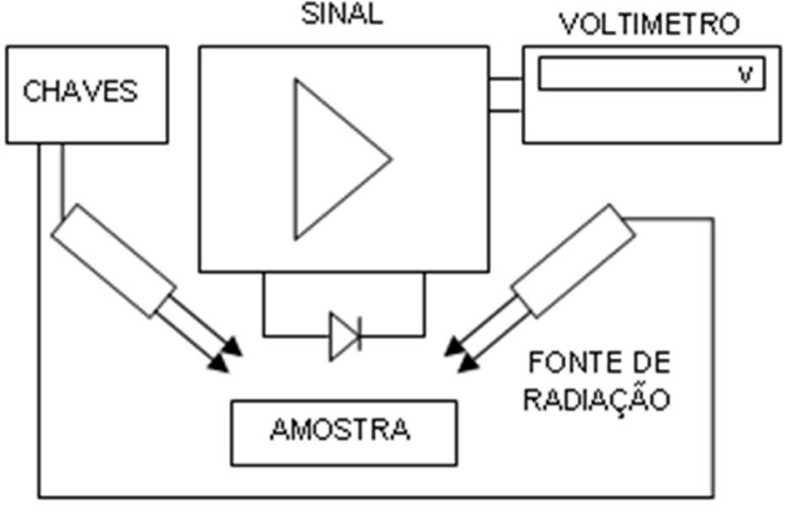

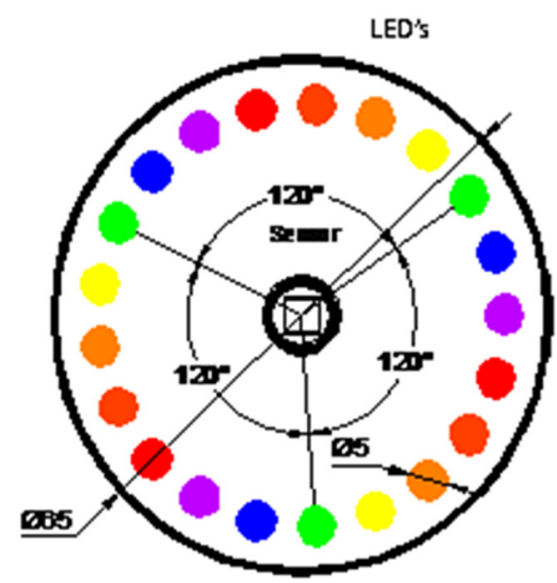

Figura 3. Diagrama de blocos do colorímetro e disposição do conjunto de LED's.

As amostras foram colocadas em um porta-amostra, a uma distância fixa da fonte de radiação e do sensor. Ao acionar a fonte, a resposta do sensor, proporcional à parte da energia radiante refletida, ao ser amplificada, foi lida em um voltímetro digital UNI-T modelo DT839B. Os dados coletados foram relacionados aos valores obtidos com o padrão de branco composto por hidróxido de magnésio prensado e depois, usados para construção das curvas e cálculo das coordenadas cromáticas.

A metodologia empregada para obtenção da refletância está de acordo com CREDER (2002), onde se supõe que a energia refletida por uma superfície branca e uniforme é aproximadamente igual à energia incidente sobre a mesma; desta forma, as refletâncias foram calculadas pela razão entre os valores obtidos com o colorímetro sobre as amostras e os valores obtidos com o colorímetro sobre a amostra de magnésio prensado (Equação 1).

$$
R_{(\%)}=100 \frac{V_{S}}{V_{P}}
$$

Em que:

$\mathrm{V}_{\mathrm{S}}$ - tensão de saída obtida nos terminais do sensor para uma amostra qualquer;

$\mathrm{V}_{\mathrm{p}}$ - tensão de saída obtida nos terminais do sensor para o padrão de branco.

Testes iniciais do colorímetro

Estudou-se a linearidade do sensor pela aplicação de estímulos luminosos variáveis; para tanto, variou-se a corrente elétrica sobre os LED's de cada cor e se mediu a iluminância do conjunto com luxímetro digital modelo TES-1332. Analisaram-se os dados coletados com o software Statistica versão 5.0 a fim de se encontrar um ajuste adequado entre a corrente elétrica e a iluminância. Repetiu-se o 
procedimento fazendo-se, porém, a medição da corrente elétrica e da resposta do sensor. Utilizaram-se os novos dados de corrente elétrica nas equações obtidas no ajuste anterior de modo que os dados estimados de iluminância pudessem ser relacionados com a resposta do sensor.

Obteve-se a variação que ocorre na resposta do sensor aplicando-se uma luminosidade constante para cada cor e fazendo-se a leitura em intervalos regulares sobre a amostra de magnésio (padrão de branco $100 \%$ de refletância) durante o período de $1 \mathrm{~h}$. Fizeram-se três repetições. O valor estável para cada cor ( $t=1 \mathrm{~h}$ ) foi usado como valor padrão no cálculo da refletância.

A metodologia adotada para comparação dos valores obtidos com o colorímetro construído e com o modelo CR10 consistiu em se calcular os valores da diferença de cor $\Delta \mathrm{E}^{*}$ ab para as 60 amostras de pó colorido e encontrar uma relação entre os dois equipamentos, pela aplicação da seguinte transformação (Equação 2 e 3):

$$
\left[\begin{array}{ll}
L^{*} & a * \\
* *
\end{array}\right]\left[\begin{array}{lll}
x_{1} & x_{2} & x_{3} \\
y_{1} & y_{2} & y_{3} \\
z_{1} & z_{2} & z_{3}
\end{array}\right]=\left[\begin{array}{lll}
L^{* \prime} & a^{* \prime} & b^{* \prime}
\end{array}\right]
$$

OU

$$
\left\{\begin{array}{l}
L^{* \prime}=L^{*} \cdot x_{1}+a^{*} \cdot y_{1}+b^{*} \cdot z_{1} \\
a^{* \prime}=L^{*} \cdot x_{2}+a^{*} \cdot y_{2}+b^{*} \cdot z_{2} \\
b^{* \prime}=L^{*} \cdot x_{3}+a^{*} \cdot y_{3}+b^{*} \cdot z_{3}
\end{array}\right.
$$

Em que:

$L^{*} a^{*} b^{*}$ são os valores encontrados com o colorímetro construído;

$L^{*} a^{*} b$ '' são os valores encontrados com o modelo CR-10;

$x_{i^{\prime}} \quad y_{i^{\prime}} \quad$ e $\quad z_{i}$ são as constantes de transformação.

Desta forma, a matriz encontrada pode ser usada como fator de correção para que os dois aparelhos forneçam dados aproximados.

Preparação dos padrões de cor

Para realização de testes iniciais com o colorímetro produziram-se padrões de cor (amostras coloridas), elaborados pela incorporação de pigmentos a um substrato branco, seguindo proporções fixas. Como substrato foram utilizados $3 \mathrm{~kg}$ de pó químico branco e seco (bicarbonato de sódio), os quais foram divididos em 60 amostras de $50 \mathrm{~g}$.

Empregou-se 1,5 $\mathrm{dm}^{3}$ de pigmento dividido em três partes de $500 \mathrm{ml}$ nas cores magenta (M), amarela (Y) e ciano (C). Os pigmentos foram misturados entre si, obtendose 30 soluções de $40 \mathrm{ml}$, com variações de 10\% dois a dois, ou seja, a amostra 1 possui 100\% do pigmento ciano (C100), enquanto as amostras 2 e 3 apresentam 90 e 10\% (C90Y10) e 80 e $20 \%$ (C80Y20) dos pigmentos ciano e amarelo, respectivamente, e sucessivamente até a amostra 30 (M10C90) composta de 10 e 90\% dos pigmentos magenta e ciano, respectivamente. As soluções de $40 \mathrm{ml}$ foram misturadas com o pó seco na proporção de $20 \mathrm{ml} / 50 \mathrm{~g}$. Das amostras obtidas, 30 foram secas em estufa a $105 \pm 1{ }^{\circ} \mathrm{C}$ e 30 a temperatura ambiente, obtendo-se 60 amostras de cores diferentes.

\section{Medidas de cor}

As cores das 60 amostras do pó colorido artificialmente foram determinadas com 0 colorímetro experimental, como função da refletância de uma superfície padrão. Obteve-se esta superfície a partir do hidróxido de magnésio prensado cuja leitura se associou a $100 \%$ de refletância. A iluminância para cada cor foi medida com luxímetro e associada à potência espectral relativa a cada cor. As constantes $\bar{x}$ , $\bar{y}$ e $\bar{z}$ associadas a cada comprimento de onda, estão relacionadas na Tabela 2.

As Equações 5 a 8 foram adaptadas para o caso discreto (Equações 4 a 7) e usadas para obtenção dos valores tristimulus de cada amostra. Os dados foram convertidos para 0 sistema de cor L*a*b* com uso das equações desse sistema como função dos valores tristimulus e comparados com os valores obtidos com colorímetro comercial, que fornece os resultados nas coordenadas deste sistema. As equações do sistema XYZ adaptadas são descritas a seguir: 
Tabela 2. Valores padronizados de $\bar{x}, \bar{y}$ e $\bar{z}$ a serem usados no cálculo dos valores tristimulus XYZ.

\begin{tabular}{cccccccc}
\hline $\mathbf{n m})$ & $\begin{array}{c}\mathbf{4 0 5} \\
\text { Violeta }\end{array}$ & $\begin{array}{c}\mathbf{4 7 0} \\
\text { Azul }\end{array}$ & $\begin{array}{c}\mathbf{5 2 5} \\
\text { Verde }\end{array}$ & $\begin{array}{c}\mathbf{5 9 0} \\
\text { Amarelo }\end{array}$ & $\begin{array}{c}\mathbf{6 0 5} \\
\text { Laranja }\end{array}$ & $\begin{array}{c}\mathbf{6 1 5} \\
\text { Âmbar }\end{array}$ & $\begin{array}{c}\mathbf{6 2 5} \\
\text { Vermelho }\end{array}$ \\
\hline $\bar{x}$ & 0,0232 & 0,1954 & 0,1096 & 1,0263 & 1,0456 & 0,9384 & 0,7514 \\
$\bar{y}$ & 0,0006 & 0,0910 & 0,7932 & 0,7570 & 0,5668 & 0,4412 & 0,3210 \\
$\bar{z}$ & 0,1102 & 1,2876 & 0,0573 & 0,0011 & 0,0006 & 0,0002 & 0,0001 \\
\hline Fonte: Moreira (1982) & & & & & & &
\end{tabular}

$$
\begin{aligned}
& \mathrm{X}=\mathrm{K} \sum_{1}^{7} \mathrm{~S}_{\mathrm{i}}(\lambda) \mathrm{R}_{\mathrm{i}}(\lambda) \overline{\mathrm{x}_{\mathrm{i}}} \\
& \mathrm{Y}=\mathrm{K} \sum_{1}^{7} \mathrm{~S}_{\mathrm{i}}(\lambda) \mathrm{R}_{\mathrm{i}}(\lambda) \overline{\mathrm{y}_{\mathrm{i}}} \\
& \mathrm{Z}=\mathrm{K} \sum_{1}^{7} \mathrm{~S}_{\mathrm{i}}(\lambda) \mathrm{R}_{\mathrm{i}}(\lambda) \overline{\mathrm{z}_{\mathrm{i}}} \\
& K=\frac{100}{\sum_{1}^{7} S_{i}(\lambda) \overline{y_{i}}}
\end{aligned}
$$

Em que:

i - índice que indica cada cor (violeta, azul, verde, amarela, laranja, âmbar e vermelha);

$\lambda$ - comprimento de onda da região visível do espectro eletromagnético (de 380 a $780 \mathrm{~nm}$ segundo a Tabela 2); função de $\bar{y}$ :

K - fator de normalização de cor em

$S(\lambda)$ - distribuição espectral de potência relativa do iluminante;

$R(\lambda)$ - refletância espectral relativa do objeto;

$\bar{x}, \bar{y}$ e $\bar{z}$ - valores tristimulus de igual energia do espectro em função de $\lambda$ para um observador padrão, segundo a Tabela 2.

As amostras em pó (padrões de cor) foram acondicionadas no porta-amostra e niveladas com uma régua de vidro, de forma a evitar o máximo de irregularidades na superfície. As medições foram tomadas a cada $30 \mathrm{~s} \mathrm{em}$ triplicata, a fim de evitar qualquer efeito de instabilidade. A cada repetição a amostra foi retirada e recolocada no equipamento. O porta-amostra tem tamanho fixo, com forma de tronco de cone e volume $10 \mathrm{ml}$, sendo que este era colocado no centro da parte interna do colorímetro, a $5 \mathrm{~mm}$ de distância da área radiante. $O$ aparelho era fechado e se iniciava o processo de obtenção dos dados.

\section{Resultados e Discussão}

\section{Características do sensor}

Os dados obtidos de iluminância em função da corrente elétrica sobre os LED's podem ser observados na Tabela 3. Tem-se, na Tabela 4, as equações de regressão da iluminância dos LED's de cada cor, em função da corrente elétrica, e os respectivos coeficientes de determinação e desvios percentuais médios. Pela boa adequação dos ajustes $\left(R^{2}>99 \%\right.$ e $P$ $<5 \%$ ), essas equações podem ser usadas para estimar a iluminância como função da corrente elétrica aplicada em cada LED.

Apresentam-se, na Tabela 5, os valores de tensão obtidos nos terminais do sensor, em volts, como função da corrente elétrica aplicada e iluminância calculada, utilizando-se as equações da Tabela 4.

Para os valores experimentais e calculados da Tabela 5, aplicou-se análise de regressão linear e quadrática. Obtiveramse, para os dois modelos, coeficientes de determinação superiores a 98,8\%, porém para - modelo linear foram obtidos desvios médios percentuais menores que $5 \%$, enquanto para o modelo quadrático os valores foram superiores a $5 \%$ para a cor azul e $10 \%$ para a cor vermelha.

Expõe-se, na Tabela 6, as equações de regressão da resposta do sensor, em função da iluminância calculada pela corrente elétrica, os respectivos coeficientes de determinação e os desvios percentuais médios para ajuste por equação linear.

\section{Velocidade de resposta do sensor}

Na Tabela 7 estão relacionados os dados da tensão média de saída do sensor em função do tempo e da cor aplicada para uma amostra de magnésio prensado. Observa-se que a maior variação da resposta do sensor se deu nos LED's 
das cores violeta, amarelo e laranja. Para o LED violeta, a diferença entre os valores obtidos foi $0,25 \mathrm{~V}$ relativamente aos tempos $5 \mathrm{~s}$ e $1 \mathrm{~h}$, o que corresponde a uma variação de $13,51 \%$ da leitura inicial; da mesma forma, para o LED amarelo a diferença encontrada foi $0,14 \mathrm{~V}$ (o que corresponde a $6,36 \%$ do valor inicial) e para o LED laranja a diferença foi $0,10 \mathrm{~V}$ (referente a $4,63 \%$ do valor inicial). Para os demais LED's as diferenças entre os valores inicial e final foram: $0,01 \vee(0,22 \%)$ para o LED azul, $0,07 \vee(1,68 \%)$ para o LED verde, $0,05 \vee(1,01 \%)$ para o LED âmbar e $0,03 \vee(0,75 \%)$ para o LED vermelho. Nota-se que a menor variação ocorreu para os LED's emissores das três cores primárias, azul, verde e vermelho, às quais o olho humano é sensivel (a cor âmbar tem comprimento de onda pouco menor que a cor vermelha, razão por que a resposta do sensor a essas duas cores foi semelhante); este comportamento pode ser explicado pelo fato de que o sensor é dotado de um filtro para correção de cor de alta qualidade, que torna sua resposta aproximada à do olho humano; esta característica faz este dispositivo ser ideal para aplicações em fotometria.

Tabela 3. lluminância dos LED's (lux) de cada cor em função da corrente elétrica (mA) aplicada.

\begin{tabular}{|c|c|c|c|c|c|c|c|c|c|c|c|c|c|}
\hline \multicolumn{2}{|c|}{ Violeta } & \multicolumn{2}{|c|}{ Azul } & \multicolumn{2}{|c|}{ Verde } & \multicolumn{2}{|c|}{ Amarelo } & \multicolumn{2}{|c|}{ Laranja } & \multicolumn{2}{|c|}{ Âmbar } & \multicolumn{2}{|c|}{ Vermelho } \\
\hline $\mathrm{i}(\mathrm{mA})$ & L (lux) & $\mathrm{i}(\mathrm{mA})$ & L (lux) & $\mathrm{i}(\mathrm{mA})$ & L (lux) & $\mathrm{i}(\mathrm{mA})$ & L (IUx) & $\mathrm{i}(\mathrm{mA})$ & L (lux) & $\mathrm{i}(\mathrm{mA})$ & L (lux) & $\mathrm{i}(\mathrm{mA})$ & L (lux) \\
\hline 21,90 & 134,5 & 21,0 & 1774 & 23,28 & 2070 & 21,40 & 507 & 22,20 & 316 & 22,07 & 537 & 21,2 & 263 \\
\hline 16,77 & 107,3 & 15,22 & 1404 & 18,00 & 1720 & 17,43 & 410 & 16,00 & 234 & 16,53 & 409 & 17,0 & 209 \\
\hline 15,25 & 98,4 & 13,63 & 1293 & 16,00 & 1570 & 15,50 & 366 & 14,55 & 215 & 14,50 & 361 & 15,0 & 184 \\
\hline 10,56 & 67,9 & 7,40 & 804 & 10,00 & 1113 & 9,00 & 210 & 7,43 & 110 & 9,00 & 226 & 9,0 & 106 \\
\hline 9,35 & 59,7 & 6,60 & 734 & 8,00 & 927 & 6,00 & 133 & 5,50 & 80 & 7,00 & 174 & 7,0 & 80 \\
\hline 8,22 & 52,0 & 4,94 & 576 & 5,60 & 690 & 5,00 & 109 & 4,10 & 57 & 5,00 & 123 & 5,0 & 54 \\
\hline 7,25 & 45,0 & 3,78 & 459 & 4,50 & 574 & 3,50 & 71 & 3,00 & 41 & 3,50 & 84 & 3,1 & 29 \\
\hline 3,03 & 14,7 & 0,44 & 53 & 0,50 & 68 & - & - & - & - & - & - & - & - \\
\hline 2,51 & 11,3 & - & - & - & - & - & - & - & - & - & - & - & - \\
\hline 1,43 & 4,9 & - & - & - & - & - & - & - & - & - & - & - & - \\
\hline
\end{tabular}

Tabela 4. Equações obtidas a partir de ajuste quadrático aos dados experimentais da iluminância em função da corrente elétrica para os LED's de cada cor.

\begin{tabular}{|c|c|c|c|}
\hline LED & Equação estimada & $\begin{array}{c}\mathbf{R}^{2} \\
(\%)\end{array}$ & $\begin{array}{c}P \\
(\%)\end{array}$ \\
\hline Violeta & $L=-0,0573 . i^{2}+7,8235 . i-8,2244$ & 99,96 & 3,69 \\
\hline Azul & $L=-1,6363 . i^{2}+116,9190 . i+21,3149$ & 99,93 & 5,48 \\
\hline Verde & $L=-1,5375 . i^{2}+122,1030 . i+34,1992$ & 99,93 & 4,67 \\
\hline Amarelo & $L=-0,0296 . i^{2}+24,9112 . i-13,7570$ & 99,99 & 2,60 \\
\hline Laranja & $L=-0,0809 . i^{2}+16,4192 . i-7,0587$ & 99,98 & 2,18 \\
\hline Âmbar & $L=-0,1030 . i^{2}+26,9759 . i-8,3535$ & 99,99 & 0,98 \\
\hline Vermelho & $L=0,00748 . i^{2}+12,6591 . i-8,5183$ & 99,98 & 4,45 \\
\hline
\end{tabular}


Tabela 5. Tensão nos terminais do sensor $\left(V_{s}\right)$ em resposta à variação da corrente elétrica (I), e iluminância calculada ( $\left.\mathrm{L}_{\text {cal }}\right)$, dos LED's de cada cor.

\begin{tabular}{|c|c|c|c|c|c|c|c|c|c|c|c|}
\hline \multicolumn{3}{|c|}{ Violeta } & \multicolumn{3}{|c|}{ Azul } & \multicolumn{3}{|c|}{ Verde } & \multicolumn{3}{|c|}{ Amarelo } \\
\hline I (mA) & $\begin{array}{l}\mathrm{L}_{\text {cal }} \\
\text { (lux) }\end{array}$ & $V_{s}(V)$ & I (mA) & $\mathrm{L}_{\text {cal }}(\mathrm{lux})$ & $V_{s}(V)$ & $I(m A)$ & $\mathrm{L}_{\text {cal }}($ lux) & $V_{s}(V)$ & I (mA) & $\begin{array}{l}\mathrm{L}_{\text {cal }} \\
\text { (lux) }\end{array}$ & $V_{s}(V)$ \\
\hline 21,80 & 135,63 & 2,73 & 20,60 & 1732,96 & 13,691 & 22,70 & 2008,81 & $13,69^{1}$ & 21,30 & 506,16 & 4,74 \\
\hline 18,88 & 119,10 & 2,69 & 18,72 & 1628,27 & 13,691 & 19,80 & 1849,14 & 13,70 & 18,84 & 445,24 & 4,18 \\
\hline 16,79 & 106,79 & 2,43 & 15,24 & 1400,99 & 13,691 & 18,13 & 1744,62 & 13,43 & 17,40 & 409,82 & 3,90 \\
\hline 15,30 & 97,76 & 1,96 & 13,79 & 1303,02 & $13,69^{1}$ & 16,20 & 1612,37 & 12,32 & 15,50 & 363,34 & 3,47 \\
\hline 14,00 & 89,73 & 1,86 & 11,17 & 1115,44 & 13,691 & 14,54 & 1488,81 & 11,35 & 13,10 & 305,07 & 2,91 \\
\hline 12,38 & 79,49 & 1,80 & 9,22 & 963,24 & 13,691 & 12,10 & 1290,70 & 9,82 & 11,00 & 254,48 & 2,40 \\
\hline 10,55 & 67,65 & 1,45 & 7,42 & 810,16 & 13,67 & 10,10 & 1113,68 & 8,51 & 9,00 & 206,64 & 1,96 \\
\hline 9,37 & 59,84 & 1,23 & 6,64 & 739,44 & 13,67 & 8,00 & 913,63 & 6,98 & 6,00 & 135,51 & 1,28 \\
\hline 8,22 & 52,12 & 1,07 & 4,95 & 575,84 & 10,78 & 5,60 & 667,19 & 5,16 & 5,00 & 111,97 & 1,04 \\
\hline 7,25 & 45,51 & 0,98 & 3,78 & 453,40 & 8,45 & 4,50 & 547,90 & 4,25 & 3,50 & 76,82 & 0,71 \\
\hline 6,62 & 41,17 & 0,89 & 2,94 & 360,44 & 6,68 & 3,02 & 381,11 & 2,93 & 2,00 & 41,85 & 0,38 \\
\hline 5,31 & 32,03 & 0,69 & 1,65 & 208,78 & 3,77 & 2,00 & 261,95 & 1,96 & 1,00 & 18,65 & 0,18 \\
\hline 4,57 & 26,79 & 0,59 & 1,00 & 128,04 & 2,22 & 1,50 & 202,29 & 1,47 & 0,70 & 11,70 & 0,13 \\
\hline 3,54 & 19,43 & 0,42 & 0,67 & 85,89 & 1,45 & 1,00 & 141,81 & 0,94 & 0,50 & 7,08 & 0,10 \\
\hline 3,03 & 15,74 & 0,35 & 0,44 & 56,04 & 0,90 & 0,50 & 80,50 & 0,45 & - & - & - \\
\hline \multicolumn{4}{|c|}{ Laranja } & \multicolumn{4}{|c|}{ Âmbar } & \multicolumn{4}{|c|}{ Vermelho } \\
\hline $\mathrm{I}(\mathrm{mA})$ & \multicolumn{2}{|c|}{$\mathrm{L}_{\text {cal }}$ (IUx) } & $V_{s}(V)$ & $\mathrm{I}(\mathrm{mA})$ & \multicolumn{2}{|c|}{$\mathrm{L}_{\text {cal }}$ (lux) } & $V_{s}(V)$ & $\mathrm{I}(\mathrm{mA})$ & \multicolumn{2}{|c|}{$\mathrm{L}_{\text {cal }}$ (lux) } & $V_{s}(V)$ \\
\hline 22,20 & \multicolumn{2}{|c|}{319,22} & 4,98 & 22,00 & \multicolumn{2}{|c|}{536,99} & 12,90 & 21,20 & \multicolumn{2}{|c|}{264,84} & 8,94 \\
\hline 18,64 & \multicolumn{2}{|c|}{270,68} & 4,00 & 18,16 & \multicolumn{2}{|c|}{447,26} & 10,32 & 19,10 & \multicolumn{2}{|c|}{236,31} & 7,98 \\
\hline 16,10 & \multicolumn{2}{|c|}{235,35} & 3,56 & 16,50 & \multicolumn{2}{|c|}{407,88} & 9,47 & 17,00 & \multicolumn{2}{|c|}{208,21} & 7,07 \\
\hline 14,54 & \multicolumn{2}{|c|}{213,35} & 3,20 & 14,53 & \multicolumn{2}{|c|}{360,68} & 8,34 & 15,03 & \multicolumn{2}{|c|}{182,26} & 6,26 \\
\hline 12,50 & \multicolumn{2}{|c|}{184,25} & 2,74 & 13,02 & \multicolumn{2}{|c|}{324,17} & 7,67 & 13,00 & \multicolumn{2}{|c|}{155,93} & 5,37 \\
\hline 9,50 & \multicolumn{2}{|c|}{140,77} & 2,02 & 11,00 & \multicolumn{2}{|c|}{274,86} & 6,55 & 11,00 & 130 & & 4,48 \\
\hline 7,43 & 110 & & 1,58 & 9,00 & 225 & 51 & 5,20 & 9,00 & 105 & & 3,35 \\
\hline 5,50 & 81 & & 1,20 & 7,00 & 175 & 65 & 4,06 & 7,00 & 80 & & 2,57 \\
\hline 4,10 & 60 & & 0,91 & 5,00 & 125 &, 27 & 2,88 & 5,00 & 56 & & 1,78 \\
\hline 3,00 & 43 & & 0,65 & 3,50 & 87 & 15 & 1,99 & 3,00 & 32, & & 0,99 \\
\hline 2,00 & 28 & & 0,42 & 2,00 & 48 & 74 & 1,07 & 2,00 & 20 & & 0,60 \\
\hline 1,00 & 13 & & 0,21 & 1,00 & 22 & 97 & 0,50 & 1,00 & 8, & & 0,24 \\
\hline 0,70 & 8, & & 0,15 & 0,70 & 15 & 21 & 0,30 & 0,70 & 5,2 & & 0,15 \\
\hline 0,46 & 4 & & 0,11 & 0,50 & 10 & & 0,23 & 0,51 & 2, & & 0,10 \\
\hline
\end{tabular}

Tabela 6. Equações obtidas a partir de ajuste linear aos dados experimentais da resposta do sensor, em função da iluminância calculada pela corrente sobre os LED's.

\begin{tabular}{|c|c|c|c|}
\hline LED & Equação estimada & $\mathbf{R}^{2}(\%)$ & $\mathrm{P}(\%)$ \\
\hline Violeta & $V s=0,0212 \cdot$ Lcal $+0,0119$ & 98,82 & 3,10 \\
\hline Azul & $V_{s}=0,0188 \cdot$ Lcal $-0,1508$ & 99,98 & 0,85 \\
\hline Verde & $V s=0,0076 \cdot$ Lcal $-0,0331$ & 99,93 & 4,00 \\
\hline Amarelo & $V s=0,0094 \cdot$ Lcal $+0,0076$ & 99,98 & 3,78 \\
\hline Laranja & $V_{s}=0,0152 \cdot$ Lcal $-0,0187$ & 99,84 & 4,83 \\
\hline Âmbar & $V s=0,0237 \cdot$ Lcal $-0,0697$ & 99,93 & 3,47 \\
\hline Vermelho & $V s=0,0342 \cdot$ Lcal $-0,0802$ & 99,93 & 2,98 \\
\hline
\end{tabular}

$\mathrm{V}_{\mathrm{s}}$ - Tensão de saída do fotossensor; $\mathrm{L}_{\mathrm{cal}}$ - iluminância calculada (lux) 
Tabela 7. Tensão média de saída do sensor, em volts, em função do tempo e cor aplicada para uma amostra de magnésio prensado.

\begin{tabular}{|c|c|c|c|c|c|c|c|c|c|c|c|c|c|c|c|}
\hline Tempo & Viol & Azul & Verd & Ama & Lar & Âmb & Ver & Tempo & Viol & Azul & Verd & Ama & Lar & Âmb & Verm \\
\hline 0:00:05 & 1,85 & 4,46 & 4,16 & 2,20 & 2,16 & 4,93 & 4,01 & $0: 03: 15$ & 1,65 & 4,48 & 4,21 & 2,09 & 2,06 & 4,87 & 4,00 \\
\hline $0: 00: 10$ & 1,81 & 4,46 & 4,17 & 2,18 & 2,14 & 4,92 & 4,01 & 0:03:20 & 1,65 & 4,48 & 4,21 & 2,09 & 2,06 & 4,87 & , 00 \\
\hline $0: 00: 15$ & 1,79 & 4,47 & 4,18 & 2,16 & 2,12 & 4,91 & 4,01 & $0: 03: 25$ & 1,65 & 4,48 & 4,21 & 2,09 & 2,06 & 4,87 & 4,00 \\
\hline 0:00:20 & 1,78 & 4,47 & 4,18 & 2,16 & 2,11 & 4,91 & 4,01 & 0:03:30 & 1,65 & 4,48 & 4,21 & 2,09 & 2,06 & 4,87 & 4,00 \\
\hline 0:00:25 & 1,77 & 4,47 & 4,18 & 2,15 & 2,11 & 4,90 & 4,01 & 0:03:35 & 1,65 & 4,48 & 4,21 & 2,09 & 2,06 & 4,87 & 4,00 \\
\hline 0:00:30 & 1,76 & 4,47 & 4,19 & 2,14 & 2,10 & 4,90 & 4,01 & 0:03:40 & 1,65 & 4,48 & 4,21 & 2,09 & 2,06 & 4,87 & 4,00 \\
\hline 0:00:35 & 1,75 & 4,47 & 4,19 & 2,14 & 2,10 & 4,90 & 4,01 & $0: 03: 45$ & 1,65 & 4,48 & 4,21 & 2,09 & 2,06 & 4,87 & 4,00 \\
\hline 0:00:40 & 1,74 & 4,47 & 4,19 & 2,14 & 2,10 & 4,90 & 4,00 & 0:03:50 & 1,65 & 4,48 & 4,21 & 2,09 & 2,06 & 4,87 & 4,00 \\
\hline $0: 00: 45$ & 1,73 & 4,47 & 4,19 & 2,13 & 2,09 & 4,90 & 4,00 & $0: 03: 55$ & 1,65 & 4,48 & 4,21 & 2,09 & 2,06 & 4,87 & 4,01 \\
\hline 0:00:50 & 1,73 & 4,47 & 4,19 & 2,13 & 2,09 & 4,89 & 4,00 & 0:04:00 & 1,65 & 4,48 & 4,21 & 2,09 & 2,06 & 4,87 & 4,01 \\
\hline 0:00:55 & 1,72 & 4,47 & 4,19 & 2,13 & 2,09 & 4,89 & 4,00 & 0:04:10 & 1,65 & 4,48 & 4,21 & 2,09 & 2,06 & 4,87 & 4,01 \\
\hline 0:01:00 & 1,72 & 4,47 & 4,20 & 2,12 & 2,09 & 4,89 & 4,00 & 0:04:20 & 1,64 & 4,48 & 4,21 & 2,09 & 2,06 & 4,87 & 4,01 \\
\hline 0:01:05 & 1,71 & 4,47 & 4,20 & 2,12 & 2,08 & 4,89 & 4,00 & 0:04:30 & 1,64 & 4,48 & 4,21 & 2,09 & 2,06 & 4,87 & 4,01 \\
\hline 0:01:10 & 1,71 & 4,47 & 4,20 & 2,12 & 2,08 & 4,89 & 4,00 & 0:04:40 & 1,64 & 4,48 & 4,21 & 2,08 & 2,06 & 4,87 & 4,01 \\
\hline 0:01:15 & 1,70 & 4,47 & 4,20 & 2,11 & 2,08 & 4,89 & 4,00 & 0:04:50 & 1,64 & 4,48 & 4,21 & 2,08 & 2,06 & 4,87 & 4,01 \\
\hline 0:01:20 & 1,70 & 4,47 & 4,20 & 2,11 & 2,08 & 4,88 & 4,00 & 0:05:00 & 1,64 & 4,48 & 4,21 & 2,08 & 2,06 & 4,87 & 4,01 \\
\hline 0:01:25 & 1,70 & 4,47 & 4,20 & 2,11 & 2,08 & 4,88 & 4,00 & 0:05:20 & 1,64 & 4,48 & 4,22 & 2,0 & 2,06 & 4,87 & 4,01 \\
\hline 0:01:30 & 1,69 & 4,47 & 4,20 & 2,11 & 2,08 & 4,88 & 4,00 & 0:05:40 & 1,64 & 4,48 & 4,22 & 2,08 & 2,06 & 4,87 & 4,01 \\
\hline $0: 01: 35$ & 1,69 & 4,47 & 4,20 & 2,11 & 2,07 & 4,88 & 4,00 & 0:06:00 & 1,64 & 4,48 & 4,22 & 2,08 & 2,06 & 4,87 & 4,01 \\
\hline 0:01:40 & 1,69 & 4,48 & 4,20 & 2,11 & 2,07 & 4,88 & 4,00 & 0:06:30 & 1,64 & 4,48 & 4,22 & 2,08 & 2,06 & 4,87 & 4,01 \\
\hline 0:01:45 & 1,68 & 4,48 & 4,20 & 2,11 & 2,07 & 4,88 & 4,00 & 0:07:00 & 1,63 & 4,48 & 4,22 & 2,08 & 2,06 & 4,87 & 4,01 \\
\hline 0:01:50 & 1,68 & 4,48 & 4,20 & 2,11 & 2,07 & 4,88 & 4,00 & 0:07:30 & 1,63 & 4,48 & 4,22 & 2,08 & 2,06 & 4,87 & 4,01 \\
\hline 0:01:55 & 1,68 & 4,48 & 4,20 & 2,10 & 2,07 & 4,88 & 4,00 & 0:08:00 & 1,63 & 4,48 & 4,22 & 2,08 & 2,06 & 4,87 & 4,01 \\
\hline 0:02:00 & 1,67 & 4,48 & 4,20 & 2,10 & 2,07 & 4,88 & 4,00 & 0:08:30 & 1,63 & 4,48 & 4,22 & 2,07 & 2,06 & 4,87 & 4,01 \\
\hline 0:02:05 & 1,67 & 4,48 & 4,20 & 2,10 & 2,07 & 4,88 & 4,00 & 0:09:00 & 1,62 & 4,48 & 4,22 & 2,07 & 2,06 & 4,87 & 4,01 \\
\hline 0:02:10 & 1,67 & 4,48 & 4,20 & 2,10 & 2,07 & 4,88 & 4,00 & 0:09:30 & 1,62 & 4,48 & 4,22 & 2,07 & 2,06 & 4,87 & 4,01 \\
\hline 0:02:15 & 1,67 & 4,48 & 4,21 & 2,10 & 2,07 & 4,88 & 4,00 & 0:10:00 & 1,62 & 4,48 & 4,22 & 2,07 & 2,06 & 4,87 & 4,01 \\
\hline $0: 02: 20$ & 1,67 & 4,48 & 4,21 & 2,10 & 2,07 & 4,88 & 4,00 & 0:15:00 & 1,62 & 4,48 & 4,22 & 2,06 & 2,06 & 4,87 & 4,01 \\
\hline 0:02:25 & 1,66 & 4,48 & 4,21 & 2,10 & 2,06 & 4,88 & 4,00 & 0:20:00 & 1,62 & 4,48 & 4,22 & 2,06 & 2,06 & 4,87 & 4,01 \\
\hline 0:02:30 & 1,66 & 4,48 & 4,21 & 2,10 & 2,06 & 4,88 & 4,00 & 0:25:00 & 1,61 & 4,48 & 4,22 & 2,06 & 2,05 & 4,87 & 4,02 \\
\hline $0: 02: 35$ & 1,66 & 4,48 & 4,21 & 2,10 & 2,06 & 4,88 & 4,00 & 0:30:00 & 1,61 & 4,48 & 4,22 & 2,06 & 2,05 & 4,88 & 4,02 \\
\hline 0:02:40 & 1,66 & 4,48 & 4,21 & 2,10 & 2,06 & 4,87 & 4,00 & 0:35:00 & 1,61 & 4,48 & 4,22 & 2,05 & 2,05 & 4,88 & 4,02 \\
\hline 0:02:45 & 1,66 & 4,48 & 4,21 & 2,10 & 2,06 & 4,87 & 4,00 & 0:40:00 & 1,61 & 4,48 & 4,22 & 2,05 & 2,05 & 4,88 & 4,03 \\
\hline $0: 02: 50$ & 1,66 & 4,48 & 4,21 & 2,10 & 2,06 & 4,87 & 4,00 & $0: 45: 00$ & 1,60 & 4,48 & 4,23 & 2,05 & 2,05 & 4,88 & 4,03 \\
\hline $0: 02: 55$ & 1,66 & 4,48 & 4,21 & 2,09 & 2,06 & 4,87 & 4,00 & 0:50:00 & 1,60 & 4,48 & 4,23 & 2,06 & 2,05 & 4,88 & 4,03 \\
\hline 0:03:00 & 1,66 & 4,48 & 4,21 & 2,09 & 2,06 & 4,87 & 4,00 & 0:55:00 & 1,60 & 4,47 & 4,23 & 2,06 & 2,05 & 4,88 & 4,03 \\
\hline 0:03:05 & 1,66 & 4,48 & 4,21 & 2,09 & 2,06 & 4,87 & 4,00 & 1:00:00 & 1,60 & 4,47 & 4,23 & 2,06 & 2,06 & 4,88 & 4,04 \\
\hline 0:03:10 & 1,66 & 4,48 & 4,21 & 2,09 & 2,06 & 4,87 & 4,00 & . & - & - & - & - & . & 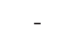 & 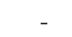 \\
\hline
\end{tabular}

Comparação das leituras de cor das amostras de pó colorido, obtidas a partir do colorímetro construído e do colorímetro Minolta CR-10

A partir dos valores da tensão média de saída do sensor obtidos para a amostra de magnésio prensado no tempo de 1 h, calcularam-se as refletâncias das 60 amostras de pó coloridas artificialmente. Os dados médios da tensão de saída do sensor para a amostra C100 (100\% do pigmento ciano) seca em estufa e para o magnésio prensado, podem ser vistos na Tabela 8. Utilizou-se a Equação 1 para o cálculo das refletâncias. Pode-se observar na, Equação 8, o modo como foi calculada a refletância correspondente à cor violeta para esta mesma amostra.

$$
R_{\text {VIOLETA }}=100 \frac{0,63}{1,60}=39,38 \%
$$

Utilizando-se as Equações de 4 a 7 e de 9 a 11 obtiveram-se os valores tristimulus XYZ e os parâmetros do espaço de cor L*a*b*, respectivamente às sessenta amostras, a partir dos dados obtidos com o colorímetro construído. Na Tabela 9 estão relacionados esses dados calculados para o colorímetro construído, das 30 amostras secas em estufa e os valores obtidos com o colorímetro Minolta CR-10ß; temse, ainda, o valor calculado com a Equação 
Tabela 8. Tensão média de saída do sensor para a amostra C100 e magnésio prensado e refletância calculada.

\begin{tabular}{c|ccccccc}
\hline LED's & Violeta & Azul & Verde & Amarelo & Laranja & Âmbar & Vermelho \\
\hline $\begin{array}{c}\text { Lensão média para o } \\
\text { magnésio (V) }\end{array}$ & 1,60 & 4,47 & 4,23 & 2,06 & 2,06 & 4,88 & 4,04 \\
$\begin{array}{c}\text { Tensão média para a } \\
\text { amostra C100 (V) }\end{array}$ & 0,63 & 1,15 & 0,73 & 0,49 & 0,60 & 1,99 & 1,99 \\
\hline Refletância calculada (\%) & 39,38 & 25,78 & 17,35 & 23,66 & 29,34 & 40,82 & 49,38 \\
\hline
\end{tabular}

12 para a diferença de cor $\Delta \mathrm{E}^{*}{ }_{\text {ab }}$ entre os dois Em que: aparelhos.

$$
\begin{aligned}
& L^{*}=116\left(\frac{Y}{Y n}\right)^{1 / 3}-16 \\
& a^{*}=500\left[\left(\frac{X}{X n}\right)^{1 / 3}-\left(\frac{Y}{Y n}\right)^{1 / 3}\right] \\
& b^{*}=200\left[\left(\frac{Y}{Y n}\right)^{1 / 3}-\left(\frac{Z}{Z n}\right)^{1 / 3}\right]
\end{aligned}
$$

$X, Y$ e Z - valores tristimulus da amostra; $\mathrm{Xn}, \mathrm{Yn}$ e $\mathrm{Zn}$ - valores tristimulus de uma superfície difusora perfeita.

$$
\Delta \mathrm{E}^{*}{ }_{\mathrm{ab}}=\sqrt{\left(\Delta \mathrm{L}^{*}\right)^{2}+(\Delta \mathrm{a} *)^{2}+\left(\Delta \mathrm{b}^{*}\right)^{2}}
$$

Em que:

$\Delta \mathrm{L}^{*}, \Delta \mathrm{a}^{*}, \Delta \mathrm{b}^{*}$, são obtidos da diferença entre cada um dos atributos $L^{*}, a^{*} e b^{*}$ dos dois

\begin{tabular}{|c|c|c|c|c|c|c|c|c|c|c|}
\hline \multirow{2}{*}{ Amostra } & \multicolumn{6}{|c|}{ Colorímetro construído } & \multicolumn{3}{|c|}{ Colorímetro Minolta CR-10 } & \multirow{2}{*}{$\Delta \mathrm{E}^{*}{ }_{\mathrm{ab}}$} \\
\hline & $\mathrm{X}$ & $\mathbf{Y}$ & $\mathbf{Z}$ & $\mathrm{L}^{*}$ & $\mathbf{a}^{*}$ & b* & $\mathrm{L}^{*}$ & $\mathbf{a}^{*}$ & b* & \\
\hline $\mathrm{Cl00}$ & 29,11 & 24,00 & 12,95 & 56,09 & 31,54 & $-1,60$ & 39,20 & 30,40 & 4,27 & 17,91 \\
\hline C90Y 10 & 32,82 & 28,92 & 11,77 & 60,71 & 25,58 & 10,34 & 41,10 & 20,23 & 19,77 & 22,41 \\
\hline C80Y20 & 37,97 & 35,71 & 13,85 & 66,30 & 19,22 & 13,17 & 46,40 & 16,63 & 25,60 & 23,61 \\
\hline C70Y30 & 34,81 & 32,00 & 9,32 & 63,34 & 21,28 & 23,98 & 44,17 & 15,83 & 33,47 & 22,08 \\
\hline C6OY 40 & 34,70 & 33,12 & 10,86 & 64,25 & 16,97 & 19,68 & 45,50 & 14,53 & 29,97 & 21,53 \\
\hline C50Y50 & 40,85 & 40,28 & 13,57 & 69,67 & 13,91 & 19,86 & 51,37 & 11,90 & 31,97 & 22,04 \\
\hline C40Y60 & 37,35 & 32,55 & 4,74 & 63,80 & 27,95 & 47,55 & 45,47 & 18,10 & 50,70 & 21,04 \\
\hline C30Y70 & 36,26 & 31,39 & 4,05 & 62,84 & 28,43 & 50,50 & 45,43 & 18,40 & 52,67 & 20,20 \\
\hline C20Y80 & 38,61 & 34,25 & 4,41 & 65,16 & 26,22 & 52,00 & 46,80 & 16,47 & 55,20 & 21,03 \\
\hline C10Y90 & 46,37 & 40,18 & 4,91 & 69,60 & 30,75 & 56,46 & 49,60 & 19,43 & 59,47 & 23,17 \\
\hline Y100 & 70,16 & 59,13 & 9,28 & 81,36 & 39,21 & 55,23 & 60,00 & 20,87 & 61,07 & 28,76 \\
\hline Y90M 10 & 37,22 & 26,66 & 4,04 & 58,66 & 49,66 & 43,35 & 39,60 & 39,63 & 40,43 & 21,73 \\
\hline Y80M20 & 33,75 & 22,33 & 3,13 & 54,37 & 56,21 & 42,93 & 35,83 & 46,20 & 37,50 & 21,76 \\
\hline Y70M30 & 31,42 & 24,06 & 7,12 & 56,15 & 40,12 & 21,29 & 39,30 & 33,13 & 25,03 & 18,62 \\
\hline Y60M40 & 30,61 & 23,27 & 7,66 & 55,35 & 40,48 & 17,37 & 37,47 & 33,17 & 21,87 & 19,84 \\
\hline Y50M50 & 27,39 & 20,66 & 7,42 & 52,58 & 39,79 & 13,69 & 36,30 & 34,37 & 18,63 & 17,86 \\
\hline Y40M60 & 23,52 & 17,87 & 7,06 & 49,33 & 37,16 & 9,81 & 34,03 & 33,50 & 16,03 & 16,92 \\
\hline Y30M70 & 19,38 & 11,90 & 3,02 & 41,06 & 52,89 & 20,91 & 28,03 & 46,53 & 20,87 & 14,50 \\
\hline Y20M80 & 22,43 & 17,15 & 9,14 & 48,44 & 35,99 & $-0,98$ & 33,13 & 33,67 & 8,93 & 18,39 \\
\hline Y10M90 & 18,16 & 13,81 & 7,06 & 43,96 & 33,97 & 0,56 & 30,47 & 33,20 & 9,33 & 16,12 \\
\hline M100 & 24,77 & 17,20 & 8,25 & 48,51 & 46,27 & 2,92 & 33,47 & 42,00 & 7,23 & 16,22 \\
\hline M90C10 & 26,29 & 18,14 & 9,40 & 49,66 & 47,79 & 0,08 & 34,27 & 42,37 & 4,47 & 16,90 \\
\hline M80C20 & 24,66 & 16,78 & 8,13 & 47,98 & 48,06 & 2,53 & 32,37 & 42,77 & 6,20 & 16,89 \\
\hline M70C30 & 22,08 & 15,43 & 7,57 & 46,22 & 43,97 & 2,03 & 31,60 & 39,80 & 5,60 & 15,61 \\
\hline M60C40 & 23,11 & 16,24 & 8,32 & 47,29 & 44,10 & 0,49 & 31,50 & 40,10 & 5,10 & 16,93 \\
\hline M50C50 & 24,02 & 18,96 & 11,98 & 50,65 & 33,77 & $-7,74$ & 36,03 & 33,07 & 1,03 & 17,06 \\
\hline M40C60 & 22,04 & 19,41 & 13,22 & 51,16 & 22,45 & $-10,96$ & 35,93 & 24,77 & 1,10 & 19,56 \\
\hline M30C70 & 26,34 & 20,93 & 12,32 & 52,87 & 34,17 & $-5,06$ & 36,80 & 31,90 & 3,43 & 18,32 \\
\hline M20C80 & 23,42 & 22,32 & 15,38 & 54,36 & 15,04 & $-11,99$ & 40,07 & 19,33 & 1,20 & 19,92 \\
\hline M10C90 & 30,47 & 22,48 & 10,48 & 54,53 & 43,52 & 4,29 & 36,47 & 35,10 & 8,13 & 20,30 \\
\hline
\end{tabular}
materiais.

Tabela 9. Valores tristimulus ( $\mathrm{X}, \mathrm{Y}$ e Z), parâmetros do espaço de cor $\mathrm{L}^{*} \mathrm{a}^{*} \mathrm{~b}^{*}$ e diferença de $\operatorname{cor}\left(\Delta \mathrm{E}^{*}\right.$ ab $)$ das 30 amostras em pó, secas em estufa, obtidos com os colorímetros construído e Minolta CR-10.

Com. Sci., Bom Jesus, v.6, n.3, p.373-384, Jul./Set. 2015 
A Figura 4 mostra a representação gráfica dos pontos da Tabela 9 com respeito ao sistema de cor L*a*b*. Percebe-se que o comportamento gráfico das cores das amostras em pó secas em estufa, para o colorímetro construído e para o modelo comercial, apresentou semelhanças.

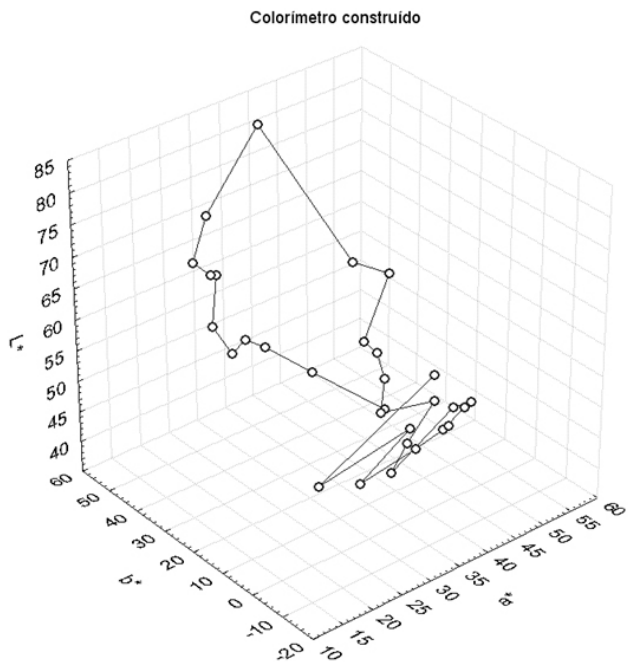

(A)

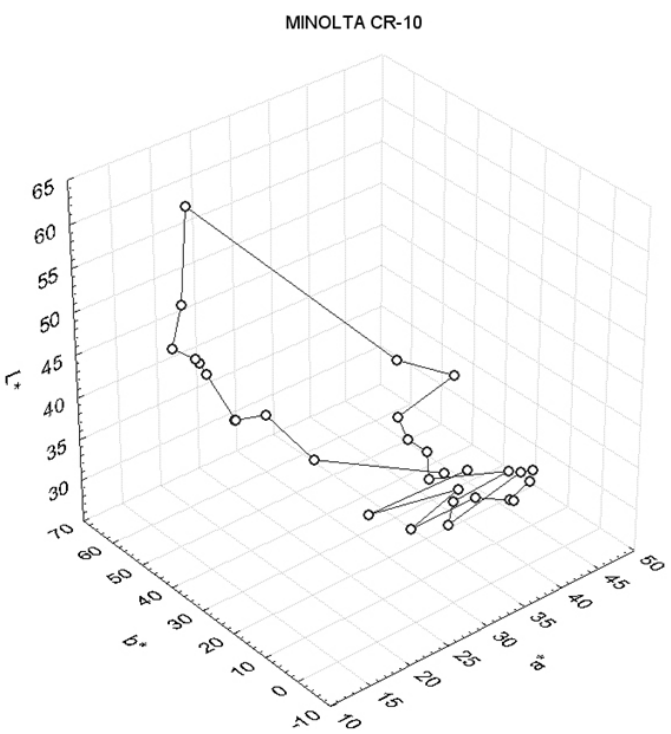

(B)

Figura 4. Valores de L*a*b* para as 30 amostras de pó secas em estufa, obtidas com colorímetro construído (A) e pelo colorímetro Minolta CR-10 (B).

De acordo com os dados analisados, observa-se que os dois equipamentos diferem significativamente, o que era esperado em virtude das características construtivas diferentes de cada um, porém como o comportamento gráfico é semelhante a relação entre os dois instrumentos foi estimada conforme a Equação 2 para os dados das 60 amostras de pó colorido, obtendo-se a seguinte matriz:

$$
\left[\begin{array}{lll}
x_{1} & x_{2} & x_{3} \\
y_{1} & y_{2} & y_{3} \\
z_{1} & z_{2} & z_{3}
\end{array}\right]=\left[\begin{array}{ccc}
0,741839 & 0,140430 & 0,312655 \\
-0,053618 & 0,749977 & -0,183716 \\
-0,021207 & -0,172033 & 0,735279
\end{array}\right]
$$

Esta é a matriz de transformação das coordenadas do sistema $L^{*} a^{*} b^{*}$ do colorímetro construído para o sistema L*a*b* do colorímetro CR-10. Foram encontrados, para a estimativa dessas constantes, coeficientes de determinação iguais a 97,96, 95,28 e 97,29\% para as colunas 1,2 e 3, respectivamente, dessa matriz. Pode-se ver, nas Figuras 5A, 5B e 5C, as relações entre os valores preditos e os observados para a estimativa dos atributos $L^{*}, a^{*} e b^{*}$, respectivamente. A equivalência encontrada entre as leituras dos dois instrumentos conferiu ao colorímetro construído uma aferição válida, visto que o padrão formado pelo colorímetro comercial utilizado é de um equipamento de uso em nível de laboratório. 


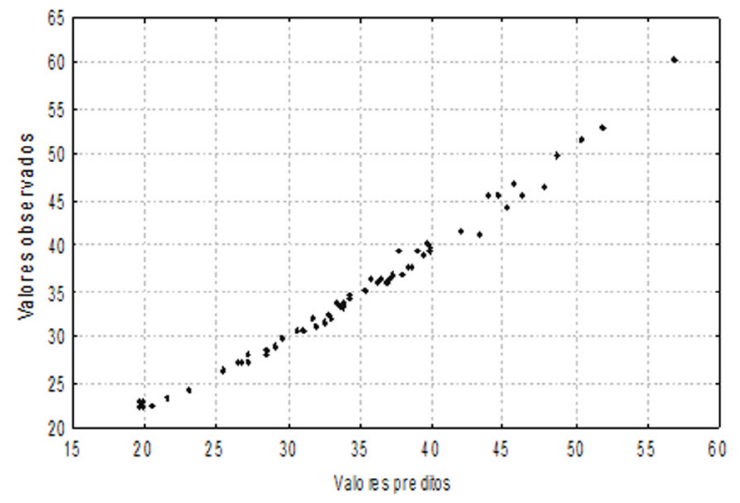

(A)

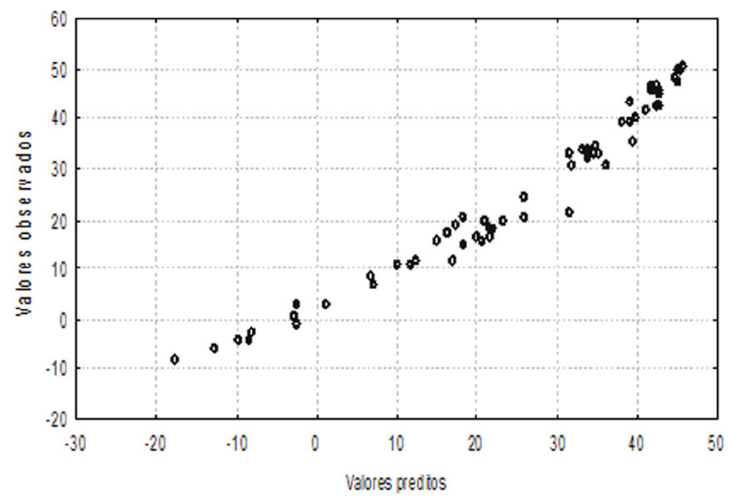

(B)

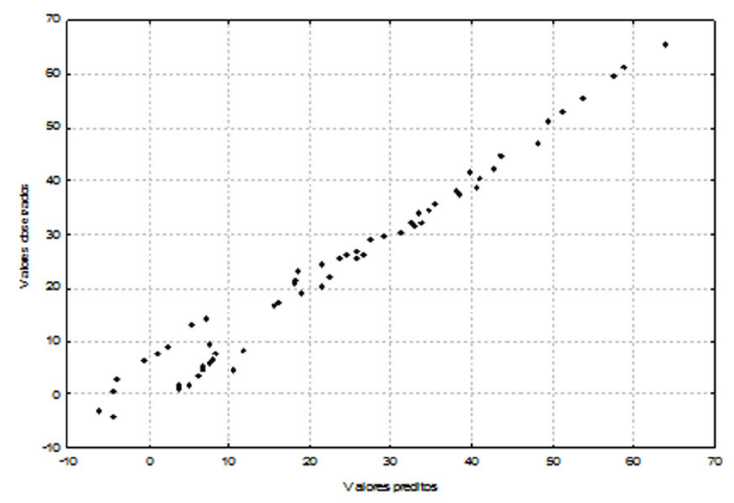

(C)

Figura 5. Relação entre valores observados e preditos na estimativa das constantes de transformação para o atributo $L^{*}(A)$, $a^{*}$ (B) e b* (C).

\section{Conclusões}

O modelo de sensor escolhido permitiu a construção de um protótipo de colorímetro de baixo custo do qual se obtiveram respostas similares às do modelo comercial.

A equivalência encontrada entre as leituras dos dois instrumentos conferiu, ao colorímetro construído, uma aferição válida.

\section{Agradecimentos}

À Coordenação de Aperfeiçoamento de Pessoal de Nível Superior (Capes) pela bolsa de mestrado.

\section{Referências}

Creder, H. 2002. Instalações elétricas. 14.ed. Livros Técnicos e Científicos, Rio de Janeiro, Brasil. $479 \mathrm{p}$.

Faraoni, A.S., RamoS, A.M., Stringheta, P.C., Laureano, J. 2008. Efeito dos métodos de conservação, tipos de embalagem e tempo de estocagem na coloração de polpa de manga "Ubá" produzida em sistema orgânico. Revista
Ceres 55: 504-511.

Leão, D.S., Peixoto, J.R., VieirA, J.V. 2006. Teor de licopeno e de sólidos solúveis totais em oito cultivares de melancia. Bioscience Journal 22: 7-15.

Luo, M.R. 2006. Applyng colour science in colour desing. Optics \& Laser Technology 38: 392-398.

Miguel, A.C.A., Albertini, S., Spoto, M.H.F. 2009. Cinética da degradação de geleiada de morango. Ciência e Tecnologia dos Alimentos 29: 142-147.

Moreira, V.A. 1982. lluminação e fotometria: teoria e aplicação. 2. ed. Edgard Blücher, São Paulo, Brasil. 196 p. 